\title{
Graphical User Interface based Signal Generator for Radar/Sonar Pulse Compression Codes
}

\author{
A NAGAJYOTHI ${ }^{1}$ \\ ${ }^{1}$ Department of ECE, Andhra University College of \\ Engineering, \\ Andhra University, 530 003, India (Research
}

Scholar)

\author{
K RAJA RAJESWARI ${ }^{2}$ \\ ${ }^{2}$ Department of ECE, Andhra University College of \\ Engineering, \\ Andhra University, 530 003, India (Professor)
}

\begin{abstract}
A high - precision and stable signal generator with extremely high frequency is ideal equipment in radar signal processing. But in radar applications there is limitation on the devices, time space and cost (in terms of value and maintenance) and is very high. To meet the demands of this special area, the paper designs such a signal generator based on Graphical User Interface in matlab which can be used for educational and research purpose. The results of the paper show that the signal generator is capable in generating various waveforms of the pulse compression codes. The performance of the codes is compared with the theoretical results which found to be accurate and can be used in wide variety of radar applications. Ambiguity Function (AF), Auto Correlation Function (ACF) and contour plots of the pulse compression codes are plotted via pop-up buttons without opening the $\mathrm{m}$-file program because of the modular design. It provides the user an option of rotating and zooming of the obtained graphs and able to calculate the performance criterion of the selected pulse compression codes
\end{abstract}

\section{Keywords}

Ambiguity Function, Auto Correlation Function, Contour plots, Pulse Compression, Discrimination (D), Figure of merit (F) Graphical User Interface (GUI and Versatile Signal Generator (VSG).

\section{INTRODUCTION}

Pulse compression allows radar to achieve the average transmitted power of a relatively long pulse, while obtaining the range resolution of short pulse. In radar where there are limitations on the peak power, pulse compression is the only means to obtain the resolution and accuracy associated with a sharp pulse but at the same time acquiring the detection capability of a long pulse. The researchers developed many pulse compression radar signals assisted by modern signal processing systems. Consequently, signals in different shapes have been presented like phase coded signals such as Barker codes, Nested Barker codes and frequency coded signals such as Simple pulse, Linear Frequency Modulation (LFM), Hyperbolic Frequency Modulation (HFM) and Costas waveform. Each of these signals has its own advantages and disadvantages .For all these codes AF, ACF and contour plots are plotted. In radar scenario, no waveform is optimum for target resolution in general. On the other hand, an optimum ambiguity surface should be of a sharp central spike surrounded by a clear area with no volume, when the bulk of the volume pushed away from the central peak, then the interference can be avoided[1-2]. The performances of these signals are stated in terms of PSLR, D, F and ISLR. This paper illustrates the design; implementation of GUI based VSG for radar/sonar pulse compression codes and is developed in Matlab ${ }^{\circledR} 7.1$.
Motivation of this paper is bring out GUI based VSG for pulse compression codes which can be used for teaching and learning process in the education and research field.

The GUI organization is done in two phases :one is design phase and another is implementation phase. The design phase involves determining the functionality of the GUI and separating the GUI into smaller controllable components. These include main window, pulse compression codes, 2D and 3D graphs of the results. The implementation stage involves realizing the functions defined in designed stage. When the simulation are completed there is an option for zooming and rotation of the obtained graphs and able to calculate the signal performance criterions of the pulse compression codes mentioned in the GUI. According to mathworks, a GUI is a practical interface to a program. A good GUI can make programs easier with a consistent appearance and with intuitive controls like pushbuttons, pop-up buttons, list boxes and so forth [5].

\section{METHODOLOGY FOR DEVELOPING THE GUI}

A GUI provides a familiar environment to work with. The GUI tool allows selecting and aligning the GUI components and to be placed in the GUI layout. Once the components are in place, there is a possibility of editing their properties: name, color, size, font, text to display and so forth. When guide saves the GUI, it creates working problem including skeleton function where the modification for implementing the behavior of the GUI is performed.

Methodology for developing the GUI based VSG for radar/sonar pulse compression codes is described in the flow chart given in Fig. 1.Typical stages of creating a GUI includes designing the GUI, laying out the GUI (Using the Layout Editor), programming the GUI (writing callbacks in M file Editor), Saving and Running the GUI. Developing a GUI begins by creating a figure with file name fig.file, by tying 'Guide' at command window or by clicking Guide symbol. Addition of components into the GUI is done by dragging the intuitive controls from the component palette. GUI can be run by the Run from the Tools menu. Guide stores a GUI in two files, which are generated for the first time. The M-file with extension.m that contains the code that controls the GUI and includes the callbacks for its components. A callback is a sequence of commands that are executable when a graphics object is activated. Here programming is done and any error found are corrected [6]. 


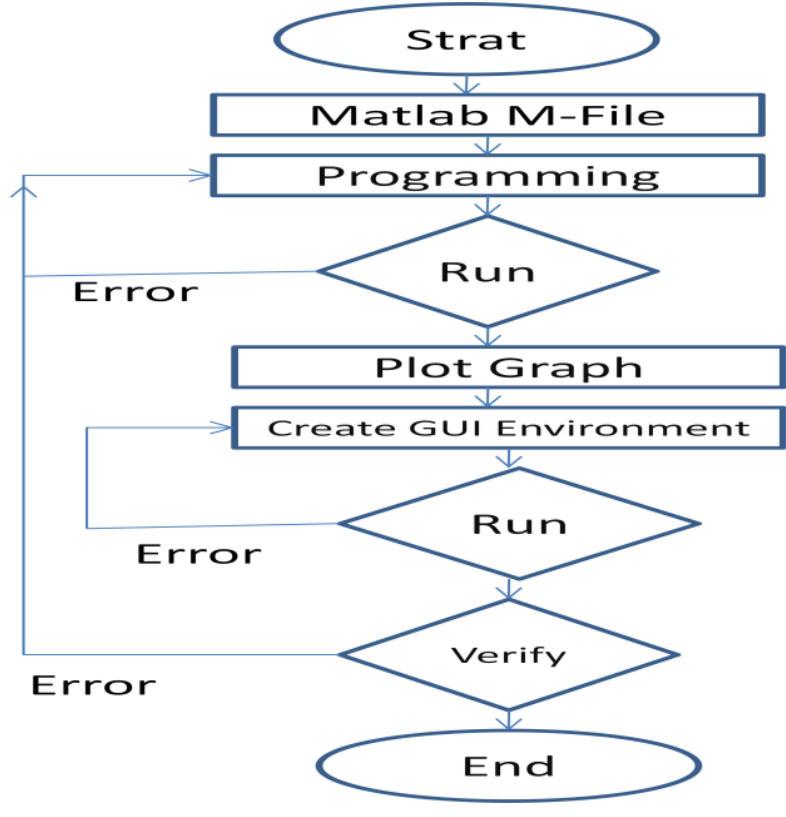

Figure. 1 Methodology for GUI based VSG for radar/sonar pulse compression codes

This flow chart describes the design procedure followed in the development of GUI. The purpose of this phase is to determine the main components of the systems so that they can be implemented individually. It gives the overall interaction between the GUI and user. The overall architecture in Fig.1 is highly abstracted, and allows easy understanding of the GUI functionality [5].

\section{AMBIGUITY FUNCTION}

In radar systems the received signal is considered as delayed version of the transmitter signal which is clearly illustrated in Fig 2. The transmitted signal is $x(t)$ and received signal is $\mathrm{x}(\mathrm{t}+\tau)$ where $\tau$ is the delay [3-4]. The cross correlation function determines the dependence of the values of one signal on another signal. For continuous signals, the output of matched filter is considered as cross-correlation function of the received signal and transmitted signal, as given in Eq.(1)

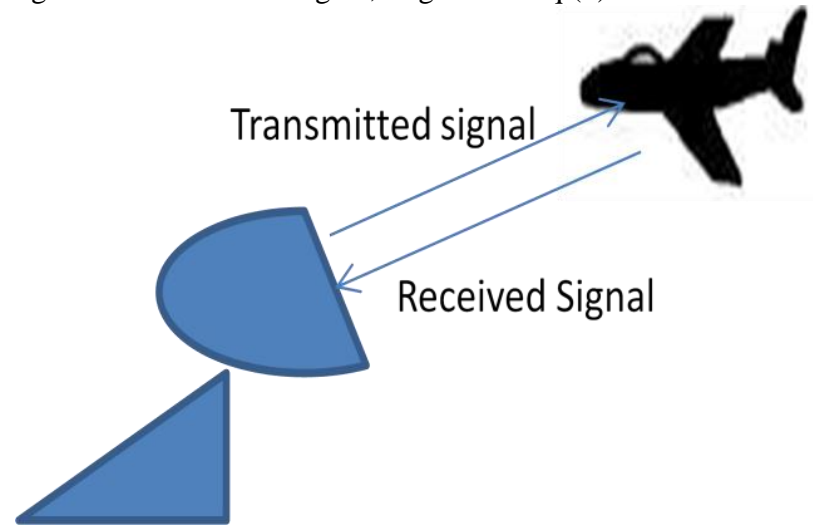

Figure 2. Transmitted and received signal in radar scenario

$$
\gamma_{x y}(t)=\int_{-\infty}^{\infty} x(t) x(t+\tau) d \tau
$$

The main aim of $\mathrm{AF}$ is to determine whether the received signal from the target at given time and frequency is matched to the receiver section.

$$
\chi\left(t, f_{d}\right)=\left|\int_{-\infty}^{\infty} x(t) e^{2 \pi j f_{d} t} x^{*}(t+\tau) d t\right|^{2}
$$

The AF, $\chi\left(t, f_{d}\right)$ is frequently given as its magnitude squared . $\mathrm{AF}$ resembles the ACF in $\mathrm{Eq}(1)$ but with the inclusion of the exponential term in Doppler frequency. The peak value of the $A F$ occurs at the origin $\mathrm{t}=0, \mathrm{f}_{\mathrm{d}}=0$ and has a value $\chi(0,0)=(2 \mathrm{E})^{2}$, where $\mathrm{E}$ is the total energy in the waveform . Ambiguity diagrams are a suitable means of depicting the delay /Doppler response of a matched filter. The cut in AF at zero Doppler gives the ACF of the transmitted wave $\mathrm{x}(\mathrm{t})$ [7-11].

Table 1. Recommended waveforms of pulse compression

\begin{tabular}{|c|c|c|}
\hline S.N & Signal & Waveform \\
\hline 1 & $\begin{array}{l}\text { Simple } \\
\text { Pulse }\end{array}$ & $\begin{aligned} \mathrm{x}(\mathrm{t}) & =\frac{1}{\sqrt{\mathrm{T}}} \quad \frac{-\tau}{2} \leq \mathrm{t} \leq \frac{-\tau}{2} \\
& =0 \quad \text { otherwise }\end{aligned}$ \\
\hline 2 & LFM & $\mathrm{x}(\mathrm{t})=\cos \left(\pi \frac{\beta}{\tau} \mathrm{t}^{2}\right) 0 \leq \mathrm{t} \leq \tau$ \\
\hline 3 & HFM & $x(t)=\exp \left[-j 2 \pi \frac{f_{1} f_{h}}{\beta} T\left(\ln \left(1-\frac{\beta t}{f_{h} T}\right)\right)\right]$ \\
\hline 4 & $\begin{array}{l}\text { Barker } \\
\text { Codes }\end{array}$ & $\begin{array}{c}x(t)=\sum_{n=0}^{N-1} x_{n}\left(t-n \tau_{c}\right) \\
x(n)= \begin{cases}\exp \left(j \varphi_{n}\right) & 0 \leq t \leq \tau \\
0 & \text { otherwise }\end{cases} \end{array}$ \\
\hline 5 & $\begin{array}{l}\text { Costas } \\
\text { Code }\end{array}$ & $\begin{array}{c}x(t)=\sum_{n=0}^{N-1} x_{n}\left(t-n \tau_{c}\right) \\
x_{n}(t)= \begin{cases}\exp [j c[n](\Delta F) t] & 0 \leq t \leq \tau_{c} \\
0 & \text { otherwise }\end{cases} \end{array}$ \\
\hline
\end{tabular}
codes

Table 1 suggests input waveforms for the pulse compression codes and when these equations are used in Eq.2 AF of respected pulse compression codes can be determined.

\section{SIGNAL CANDIDATES}

A number of signals are designed for target identification. For comparison of signal performance, goodness measures are required. An ideal $\mathrm{AF}$ requires narrow central peak and low noise floor. This implies that the ratio between mainlobe peak and sidelobe peak should be as high as possible. In view of invariance property of $\mathrm{AF}$, energy removed/reduced at one place has to appear at another place. This requires that most of the energy is to be contained in the mainlobe and as less as possible in the sidelobe. Taking this principle into consideration the signal candidates are evaluated [2,8-10]. Most of the criteria given in literature are for ACF and they are;.

Discrimination (D): Discrimination (D) is defined as the ratio of main Peak in the ACF to the absolute maximum amplitude among the side lobes

$$
\mathrm{D}=\frac{\mathrm{x}(0)}{\max |\mathrm{x}(\mathrm{k})|} \quad \mathrm{k} \neq 0
$$

Merit Factor (F): Merit factor, F, is defined as the ratio of energy in the main lobe of ACF to the total signal energy in side lobes 


$$
\mathrm{MF}=\frac{\mathrm{x}^{2}(0)}{2 \sum_{\mathrm{k}=1}^{\mathrm{N}-1} \mathrm{x}^{2}(\mathrm{k})}
$$

\section{VERSATILE SIGNAL GENERATOR}

The purpose of this VSG is to generate a variety of pulse compression codes and evaluate their time-domain and frequency domain characteristics. The GUI by itself is an important contribution for future research in its area. The GUI implemented can generate the seven different pulse compression codes and they are simple pulse, LFM, HFM, Barker, Nested Barker, Welch Costas codes, Golomb Costas codes. The block diagram of the VSG is shown in Fig 1.

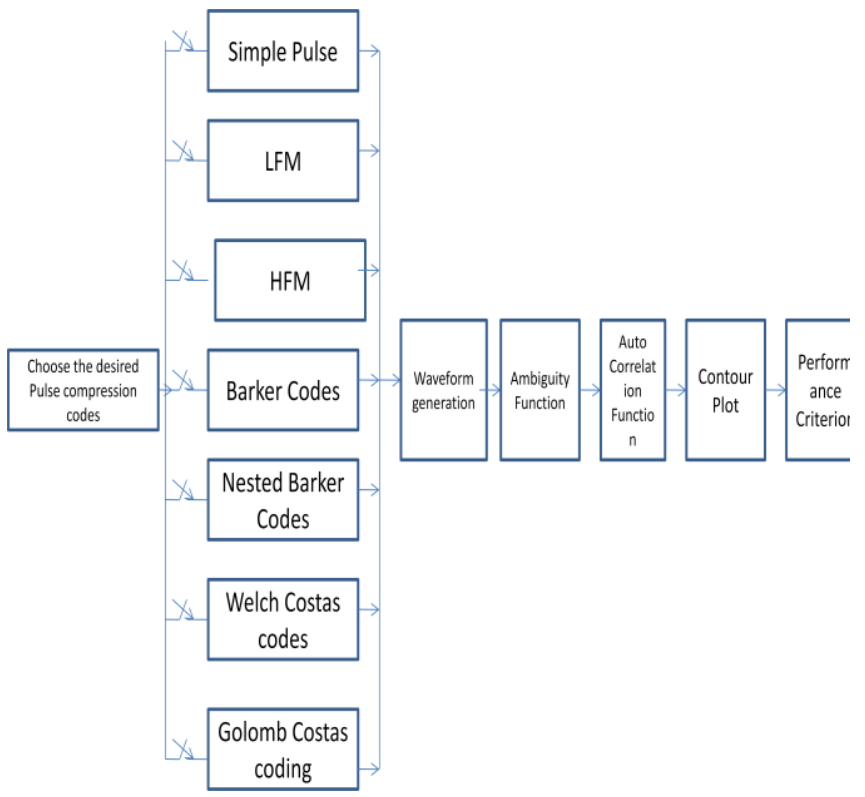

Figure 3. Block diagram of VSG

The GUI begins by creating a main window and includes all the available pulse compression codes. Because of the modular design by clicking on the respective button in the preset list, the generation of signals and their respective $\mathrm{AF}, \mathrm{ACF}$ and Contour plots are carried without opening the $\mathrm{m}$-file program. The performance criterions of each pulse compression codes are evaluated. When the program is executed it generates a GUI as shown in Figure 4.

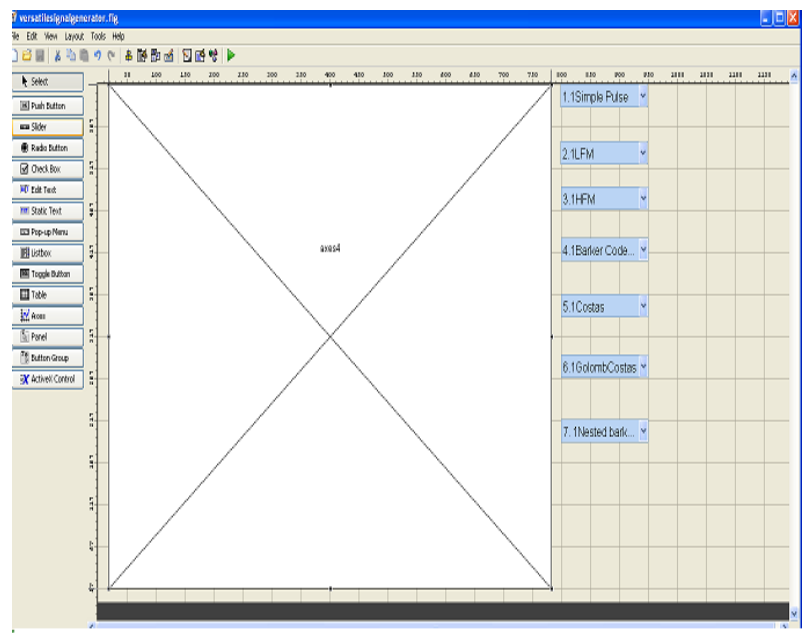

Figure 4. GUI created in Matlab

Operation of the GUI starts by selecting a function from the preset list. For example if we want to plot the AF of simple pulse ; in the GUI choose the desired signal and click on the ambiguity function of simple pulse from the popup menu. The code generated allows inputting seven different pulse compression codes and provides control over the parameters. Table 1 suggests the input waveform by which the pulse compression codes performance can be stated. In this paper by using the GUI two examples of AFs are presented. Fig.5 (corresponding to line1 in Table1) presents for simple pulse; Fig.6 (corresponding to line2 in Table1) presents for LFM; Fig.7 (corresponding to line3 in Table1) presents for HFM; Fig.8 (corresponding to line4 in Table1) presents for Barker sequence; Fig.9 (corresponding to line5 in Table1) presents for Costas Sequence; [11].

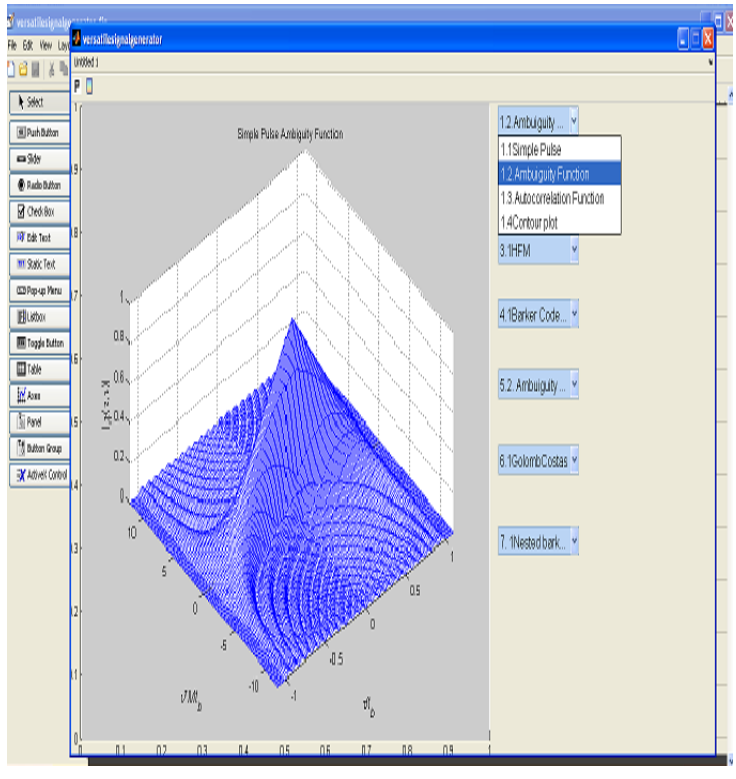

Figure 5. Ambiguity Function of Simple Pulse

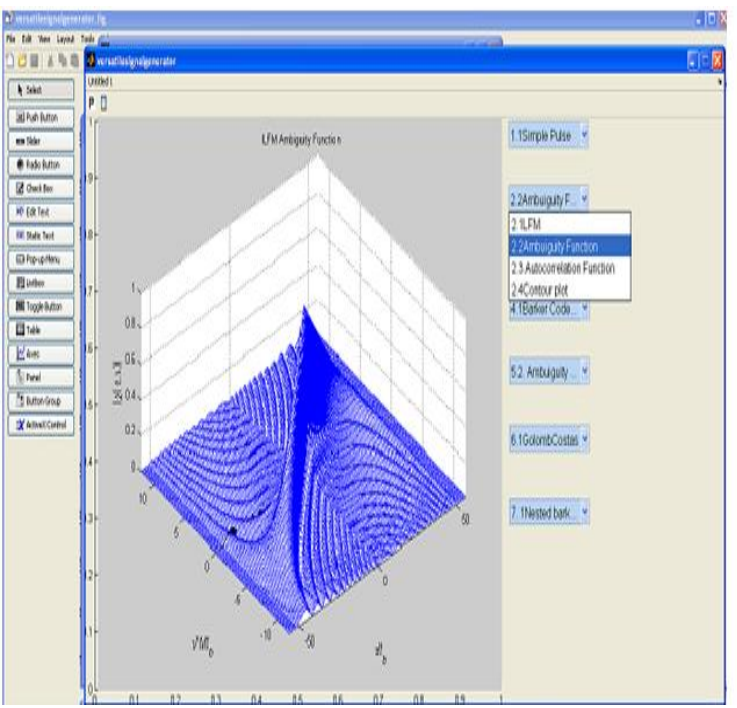

Figure.6 Ambiguity Function of a LFM 


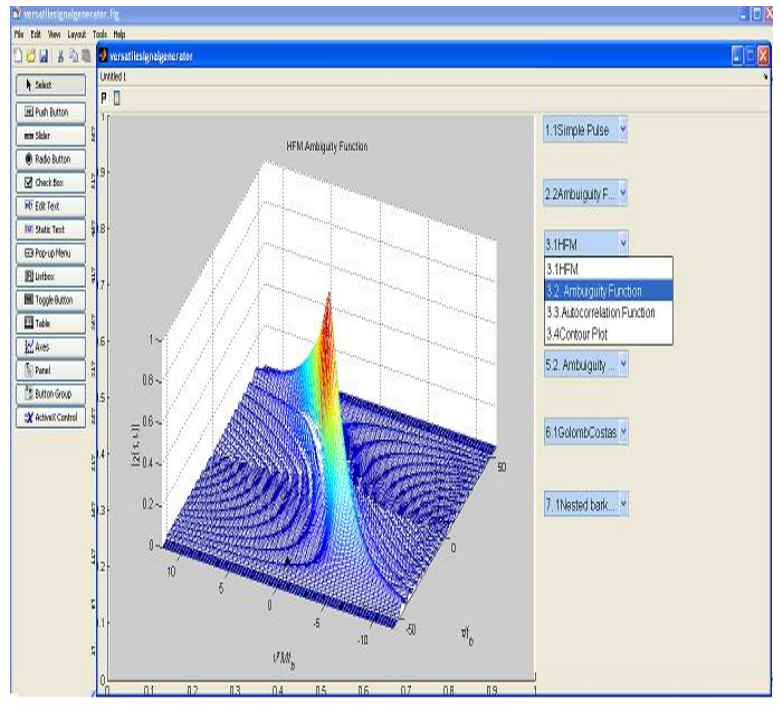

Figure 7. Ambiguity Function of HFM

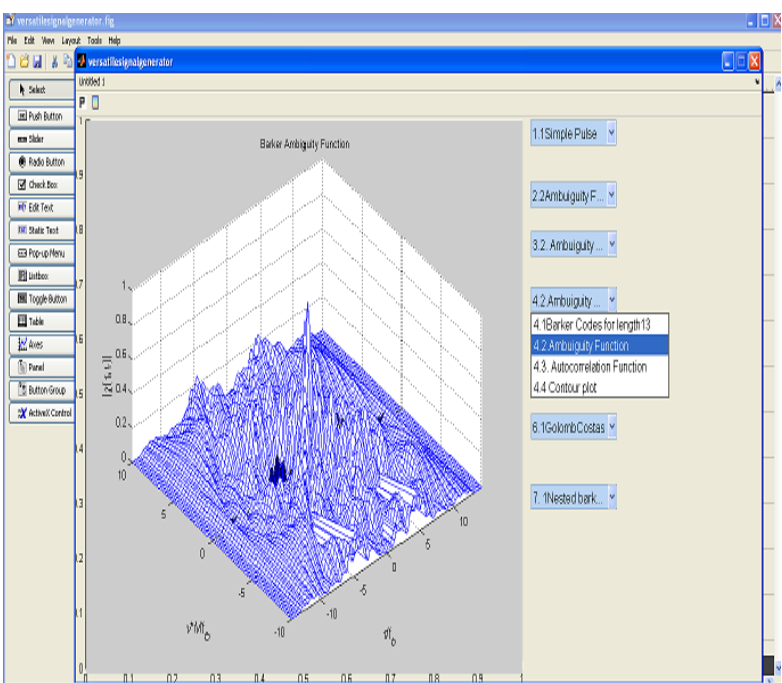

Figure. 8 Ambiguity Function of Barker code of length 13

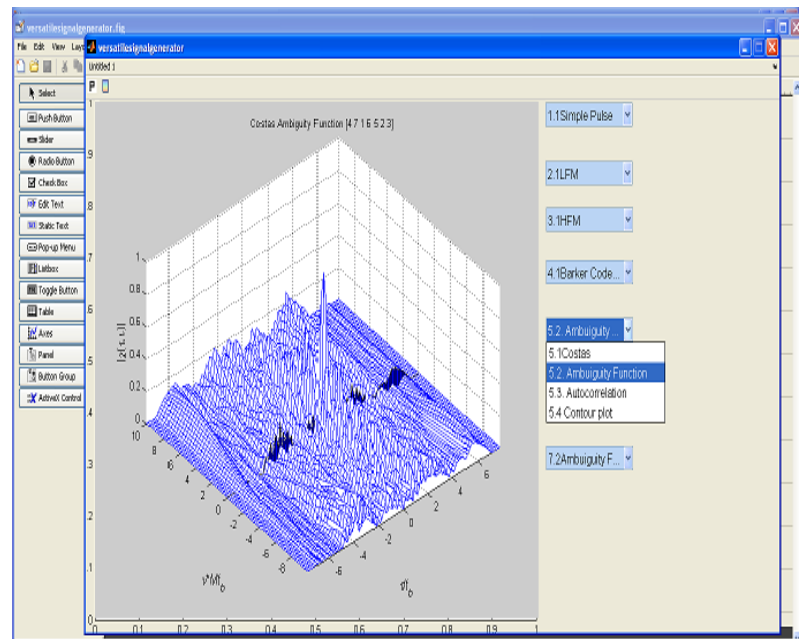

Figure.9 Ambiguity Function of Welch Costas code for $\mathrm{N}=7$

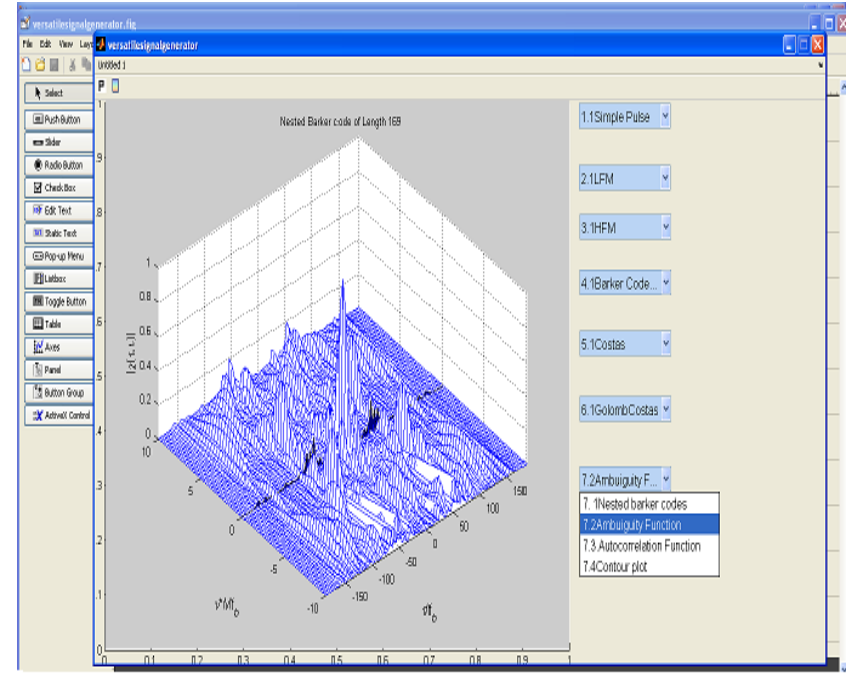

Figure.10 Ambiguity Function of Nested Barker code of length 65

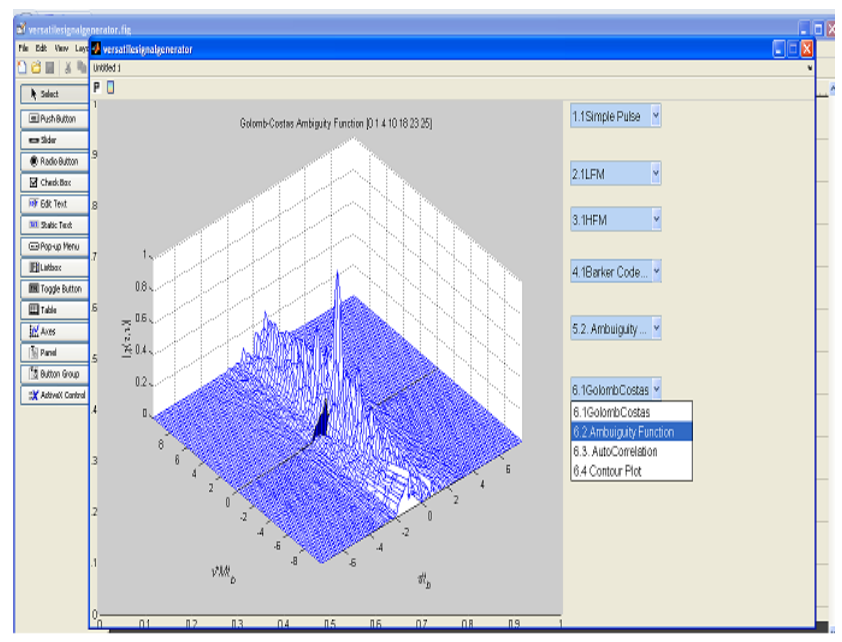

Figure.11 Ambiguity Function of Golomb Costas code for $\mathrm{N}=7$

\section{RESULTS}

Many goodness measures of the waveform are given for auto correlation domain. In this paper $\mathrm{MF}$ and $\mathrm{D}$ of pulse compression waveforms are evaluated. A GUI capable of plotting ambiguity functions of many different radar signals is presented. The GUI could be useful as a pedagogical tool in radar courses teaching pulse compression. The program also produces other figures like auto correlation functions and contour plots. The table 2 illustrates the signal performance criterions. 
Table 1. Signal performance criterions of pulse compression codes

\begin{tabular}{|l|l|l|}
\hline $\begin{array}{l}\text { Pulse Compression } \\
\text { Code }\end{array}$ & MF & D \\
\hline Simple Pulse & 1.0078 & 0.0152 \\
\hline LFM & 1.0614 & 0.1952 \\
\hline HFM & 1.12 & 0.28 \\
\hline Barker(13) & 14.08 & 13.00 \\
\hline $\begin{array}{l}\text { Nested Barker(65) } \\
\text { Kron(13,5) }\end{array}$ & 20.64 & 16.95 \\
\hline $\begin{array}{l}\text { Welch Costas(7) } \\
\text { [4 7 3 1 6 2] }\end{array}$ & 13.74 & 6.64 \\
\hline $\begin{array}{l}\text { Golomb Costas(7) } \\
\text { [0 1 4 10 18 23 25] }\end{array}$ & 27.02 & 23.58 \\
\hline
\end{tabular}

\section{CONCLUSION}

The objective of this VSG is to produce different plots showing the characteristics, facilitating understanding and analysis of the pulse compression codes. The results include AF, ACF i.e. output of matched filter and signal candidates. Validation of the GUI based VSG for radar/sonar pulse compression codes is performed by comparing the simulations results with theory. The output of the GUI found to be accurate for a wide variety of radar applications.

\section{ACKNOWLEDGMENT}

This work is being supported by Ministry of Science \& Technology, Department of Science \& Technology (DST), New Delhi, India, under Women Scientist Scheme (WOS-A) with the Grant No: 100/ (IFD)/8450/2010-11, Dated 15/11/2010.

\section{REFERENCES}

[1] Rihaczek.A.W., Principles of High Resolution Radar, McGraw-Hill, New York, 1969.

[2] Levanon. N, Mozeson Radar Signal, 2004.

[3] Bassem R. Mahafza, Radar systems analysis and design using MatLab, Chapman \& Hall/CRC, 2000

[4] P. Z. Peebles. Radar Principles. Wiley-Interscience. 1998.

[5] Flexible Extensible Radar Simulator[online] Available :http://sourceforge.net/projects/fers.

[6] M. Brooker. The Design and Implementation of a Simulator for Multistatic Radar Systems. Master's thesis, University of Cape Town, June 2008.
[7] Mark.A.richards, Fundamentals of radar signal processing, McGraw-hill, 2005

[8] NagaJyothi, A., and K. Raja Rajeswari. "Costas sequences with high PSLR.",IRACST - Engineering Science and Technology: An International Journal (ESTIJ), ISSN: 2250-3498, Vol.2, No.1, 2012

[9] NagaJyothi, A., and K. Raja Rajeswari. "Cross-correlation of Barker code and Long binary signals." International Journal of Engineering Science 3.12 (2011): 8348-8356.

[10] Jacek Marszal, Roman Salamon, Distance Measurement Errors in Silent Fm-Cw Sonar with Matched Filtering, Metrology and Measurement Systems, 2012, XIX, 2

[11] J. Yang, T.K. Sarkar, Acceleration-invariance of hyperbolic frequency modulated pulse compression, Digital Signal Processing, 2008, 18, 2, 228

\section{AUTHOR'S PROFILE}

A.NagaJyothi was born in 1982 at Visakhapatnam. She received her B.Tech (ECE) from Nagarjuna University and M.Tech(Radar \& Microwave Engineering) from Andhra University College of Engineering(A). She has a teaching experience of 3 years. Presently she is perusing her $\mathrm{Ph} . \mathrm{D}$ in the area of Signal Processing in Andhra University, Visakhapatnam.

K. Raja Rajeswari obtained her B.E., M.E. and Ph.D. degrees from Andhra University, Visakhapatnam, India in 1976, 1978 and 1992 respectively. She has published over 200 papers in various National, International Journals and conferences. She is author of the textbook Signals and Systems published by PHI. She is co-author of the textbook Electronics Devices and Circuits published by Pearson Education. Her research interests include Radar and Sonar Signal Processing, Wireless Communication Technologies.

She has guided fifteen Ph.D.s and presently she is guiding twenty students for Doctoral degree. She served as Chairperson IETE Visakhapatnam Centre for two consecutive terms (2006 t0 2010). Present she is Governing Council Member of IETE, New Delhi. She is zonal coordinate(for south) and Technical Program Committee Chairperson. She is recipient of prestigious IETE Prof SVC Aiya Memorial National Award for the year 2009, Best Researcher Awardee by Andhra University for the year 2004 and recipient Dr. Sarvepalli Radhakrishnan Best Academician Award of the year 2009 by Andhra University. She is Senior Member in IEEE. She is expert member for various national level academic and research committees and reviewer for various national/international journals. 